\title{
Fundamental theory and computer simulation of the decay characteristics of piano sound
}

\author{
Isao Nakamura \\ Department of Computer Science and Information Mathematics, \\ University of Electro-Communications \\ 1-5-1 Chofugaoka, Chofu, Tokyo, 182 Japan
}

(Received 12 May 1989)

\begin{abstract}
It is shown by theory and computer simulation that the decay characteristics of piano sound are dependent upon the degree of coupling between the strings. String and soundboard vibration are calculated using an equivalent circuit. Each string is comprised of resonance circuits that correspond to partials, and the behavior of individual partials is described independently of each other by these circuits. When the most simplified version of only one partial is considered, the equivalent circuit is expressed by the two resonant circuits coupled with the soundboard impedance. The degree of coupling between the two strings is dependent on the ratio of two constants: the degree of mistuning and the ratio of soundboard impedance to string impedance. If the former is smaller than the latter, the two strings are closely coupled with each other and a double decay characteristic results. If the relation between the two constants is reversed, the coupling is loose and a beat type decay characteristic results. The decay characteristics are changed by the above two constants. The results show that the degree of tuning and the characteristics of the strings and the soundboard determine the decay characteristics of piano sound.
\end{abstract}

PACS number: 43. 75. $\mathrm{Mn}, 43.75 . \mathrm{Wx}$

\section{INTRODUCTION}

A string starts to vibrate when a portion of the kinetic energy of a hammer is transfered to it through a hammer-string interaction. Next, the energy stored in the string gradually leaks to the soundboard, then is partly radiated into the air as sound. Since two or three strings are used for one note, except for several low notes, an exchange of energy among strings occurs through the soundboard, making the string vibrations very complex. Furthermore, strings vibrate not only in the direction normal to the soundboard but also parallel to it.

A complex decaying phenomenon of the string vibration is observed. An initial prompt sound with a large decay rate is followed by a slowly decaying after sound (Martin ${ }^{1)}$ 1947). The decay characteristic is dependent on how the two or three strings of a note are tuned to each other. When they are tuned unison, the largest decay rate is achieved. As they are more out-of-tuned, the decay gets slower. Kirk ${ }^{2)}$ (1959) says that the most favored amount of mistuning is 1 or 2 cents among strings of a note.

Weinreich $^{3,4)}(1977,1979)$ successfully explained the relation between the Martin's double decay phenomenon and the Kirk's out-of-tuning of bi- or tri-chord. Right after the hammer's impact, the string vibration decays fast since the vertical component, a main portion of the initial string vibration, has a larger decay rate than the horizontal component. After some period of time, the horizontal component dominates and hence the decay rate slows down. This type of double decay is possible for a mono-chord. In case of a bi- or tri-chord, initial vibrations of individual strings are in phase and, therefore, the decay is fast. But, gradually, the 
vibrations get out-of-tune with each other because of slight detuning among strings. This makes the vibration of the soundboard small, resulting in a slow energy leak from the strings (slow decay rate).

Hundley, Benioff and Martin ${ }^{5)}$ (1978) also discussed factors that contribute to the double decay rate. They concluded that, among five possible factors, the interaction between strings tuned close to each other was the main reason for the double decay and that the direction of the string vibration was not a factor since the vertical component dominated the vibration throughout the duration of the tone. They conducted an experimental simulation by use of a lumped electrical model.

Nakamura $^{6)}$ (1981) performed a computer simulation to study vibrations of strings for note $\mathrm{C}_{4}$. The envelope of the force acting on the bridge (simulation) and the sound pressure of an upright piano (experiment) were compared. When the lower and higher string's frequencies are set 1 cent off from the middle string's frequency, a prompt sound with a quick decay proceeds during the first 1 second, and then a slowly decaying aftersound follows.

Wogram $^{7)}$ (1981) measured the dependence of decay characteristics of piano sounds on the soundboard and found that a double decay phenomenon is observed even for a single string.

Yanagisawa et al. ${ }^{8)}$ (1984) measured velocity amplitudes of one string for note $\mathrm{C}_{3}$ when its resonance frequency (i.e., tension) is varied while the other two strings are damped. As the string resonance approaches the soundboard resonance, the decay rate increases and the double decay phenomenon is observed again for a single string vibration.

Nakamura and Iwaoka ${ }^{9}$ (1986) took into account vibrations in the horizontal direction as well as in the vertical direction and the coupling between them in their computer simulation. At the beginning, the energy of the vertical components is transfered to the horizontal components resulting in a quick decay, but once they are balanced the decay slows down. This phenomenon was suggested by Weinreich (1977 and 1979).

Meyer and Melka ${ }^{10)}$ (1983) measured decay characteristics for various notes from bass to treble. Various types of decay characteristics are observed such as linear, double, and beat decays.

Similar patterns appear in the decay characteristics of the first 14 partials for $\mathrm{G}_{1}$ in Nakamuras' study ${ }^{11}$ ) (1987).
In this paper, a simple model is employed to explain the vibration characteristics of multiple strings coupled with the soundboard for a piano key (one note) theoretically as well as by a computer simulation.

\section{EQUIVALENT ELECTRICAL CIRCUIT MODELS AND THEORETICAL COMPUTATION}

A model of a coupling system of multiple strings and a soundboard for a piano key (one note) is shown in Fig. 1. Figure 2 shows a mobility analogy equivalent electrical circuit of the model, where each string is comprised of resonance circuits that correspond to each partial, and the soundboard is assumed to have a finite impedance. The electric current and the voltage in these circuits correspond to the force and the velocity, respectively. This model simulates the inharmonicity of the piano string. It can also express that one of the three strings is in tune exactly to one note, and the other two are tuned slightly

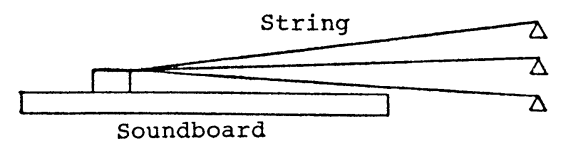

Fig. 1 Model of a string-soundboard system for a piano key (one note).

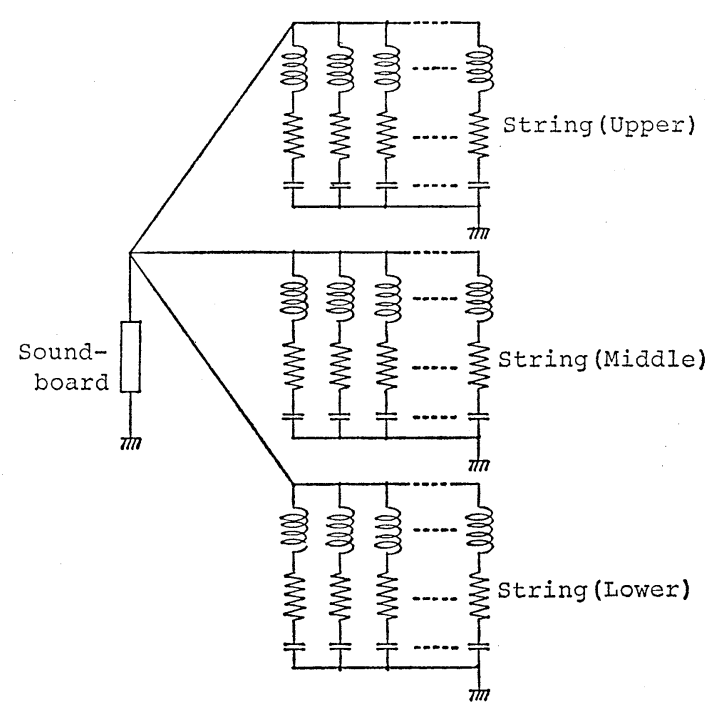

Fig. 2 Equivalent electrical circuit of the string-soundboard system. 


\section{NAKAMURA: THE DECAY CHARACTERISTICS OF PIANO SOUND}

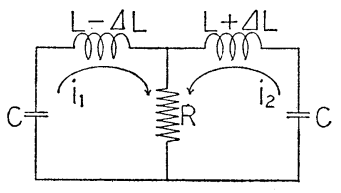

Fig. 3 Simplified version of the equivalent circuit.

lower and higher, respectively. Figure 3 shows the most simplified version of an equivalent circuit model of two strings, when the air friction and sound radiation of the string are not taken into consideration and the soundboard has a pure resistance. The difference of string stiffnesses is shown as $\Delta L$. The basic pattern of the decay characteristics of piano sound is expressed by obtaining the time variances of this coupled resonating system.

The equations of the circuit can be written as follows,

$$
\left.\begin{array}{l}
(L-\Delta L) \cdot \frac{d i_{1}}{d t}+R\left(i_{1}+i_{2}\right)+\frac{1}{C} \int i_{1} d t=0 \\
(L+\Delta L) \cdot \frac{d i_{2}}{d t}+R\left(i_{1}+i_{2}\right)+\frac{1}{C} \int i_{2} d t=0
\end{array}\right\}
$$

The initial conditions are given by

$$
i_{1}(0)=i_{2}(0)=0
$$

and when the velocity at the collision of the hammer is equivalent to the voltage $E_{\mathrm{H}}$, the charge $Q$ of the capacitor $C$ becomes

$$
Q=C E_{\mathrm{H}} \text {. }
$$

By the Laplace transformation, Eq. (1) becomes

$$
\left.\begin{array}{c}
(L-\Delta L) S I_{1}(S)+R\left\{I_{1}(S)+I_{2}(S)\right\} \\
+\frac{1}{S}\left\{\frac{1}{C} I_{1}(S)-E_{\mathrm{H}}\right\}=0 \\
(L+\Delta L) S I_{2}(S)+R\left\{I_{1}(S)+I_{2}(S)\right\} \\
+\frac{1}{S}\left\{\frac{1}{C} I_{2}(S)-E_{\mathrm{H}}\right\}=0
\end{array}\right\} .
$$

$$
\begin{aligned}
\lambda \doteqdot & \frac{1}{L C\left(1-\frac{\Delta L^{2}}{L^{2}}\right)}\left[1-\frac{1}{4}\left(\frac{\Delta L^{2}}{L^{2}}+\frac{R^{2}}{L / C}\right)\right. \\
& +\frac{1}{4} \sqrt{\left.\left(\frac{\Delta L^{2}}{L^{2}}-\frac{R^{2}}{L / C}\right)^{2}\left\{1-\left(\frac{\Delta L^{2}}{L^{2}}+\frac{R^{2}}{L / C}\right)\right\}+\frac{1}{2} \frac{\Delta L^{2}}{L^{2}} \frac{R^{2}}{L / C}\left(\frac{\Delta L^{2}}{L^{2}}+\frac{R^{2}}{L / C}\right)\right]}
\end{aligned}
$$

where When

and when

where

From Eq. (4), $I_{1}(S)$ and $I_{2}(S)$ become

$$
\left.\begin{array}{l}
\left.F(S) I_{1}(S)=S^{2} \frac{E_{\mathrm{H}}}{L\left(1-\frac{\Delta L}{L}\right)}+\frac{E_{\mathrm{H}}}{L^{2} C\left(1-\frac{\Delta L^{2}}{L^{2}}\right)}\right), \\
F(S) I_{2}(S)=S^{2} \frac{E_{\mathrm{H}}}{L\left(1+\frac{\Delta L}{L}\right)}+\frac{E_{\mathrm{H}}}{L^{2} C\left(1-\frac{\Delta L^{2}}{L^{2}}\right)}
\end{array}\right\}
$$

$$
\begin{aligned}
F(S)= & S^{4}+\frac{2 R}{L\left(1-\frac{\Delta L^{2}}{L^{2}}\right)} S^{3}+\frac{2}{L C\left(1-\frac{\Delta L^{2}}{L^{2}}\right)} S^{2} \\
& +\frac{2 R}{L^{2} C\left(1-\frac{\Delta L^{2}}{L^{2}}\right)} S+\frac{1}{L^{2} C^{2}\left(1-\frac{\Delta L^{2}}{L^{2}}\right)} \\
= & S^{4}+a S^{3}+b S^{2}+c S+d .
\end{aligned}
$$

$F(S)$ is expressed as a product of two components.

$$
\begin{aligned}
\frac{\Delta L}{L} \geq & \frac{R}{\sqrt{L / C}}, \\
F(S)= & \left\{S^{2}+\frac{1}{2}\left(a+\frac{a \lambda-c}{\sqrt{\lambda^{2}-d}}\right) S+\left(\lambda+\sqrt{\lambda^{2}-d}\right)\right\} \\
& \times\left\{S^{2}+\frac{1}{2}\left(a-\frac{a \lambda-c}{\sqrt{\lambda^{2}-d}}\right) S+\left(\lambda-\sqrt{\lambda^{2}-d}\right)\right\},
\end{aligned}
$$

$$
\begin{aligned}
\frac{\Delta L}{L} \leq & \frac{R}{\sqrt{L / C}}, \\
F(S)= & \left\{S^{2}+\frac{1}{2}\left(a+\sqrt{a^{2}+8 \lambda-4 b}\right) S\right. \\
& \left.+\left(\lambda+\frac{a \lambda-c}{\sqrt{a^{2}+8 \lambda-4 b}}\right)\right\} \\
& \times\left\{S^{2}+\frac{1}{2}\left(a-\sqrt{a^{2}+8 \lambda-4 b}\right) S\right. \\
& \left.+\left(\lambda-\frac{a \lambda-c}{\sqrt{a^{2}+8 \lambda-4 b}}\right)\right\},
\end{aligned}
$$




$$
\fallingdotseq \begin{array}{ll}
\frac{1}{L C\left(1-\frac{\Delta L^{2}}{L^{2}}\right)}\left[1-\frac{1}{2} \frac{R^{2}}{L / C}\right], & \frac{\Delta L}{L}>\frac{R}{\sqrt{L / C}}, \\
\frac{1}{L C\left(1-\frac{\Delta L^{2}}{L^{2}}\right)}\left[1-\frac{1}{2} \frac{\Delta L^{2}}{L^{2}}\right], & \frac{\Delta L}{L}<\frac{R}{\sqrt{L / C}} .
\end{array}
$$

The solution of Eq. (5) is given by the Inverse-Laplace transformation.

When $\frac{\Delta L}{L}>\frac{R}{\sqrt{L / C}}$,

$$
\left.\begin{array}{l}
i_{1}(t) \fallingdotseq \frac{E_{\mathrm{H}}}{\sqrt{L / C}}\left[\left(\sin \omega_{1} t+\frac{1}{2} \frac{R}{\sqrt{L / C}} \cdot \frac{1}{\frac{\Delta L}{L}} \cos \omega_{1} t\right) e^{-\alpha_{1} t}-\frac{1}{2} \frac{R}{\sqrt{L / C}} \cdot \frac{1}{\frac{\Delta L}{L}} \cos \omega_{2} t e^{-\alpha_{2} t}\right] \\
i_{2}(t) \fallingdotseq \frac{E_{\mathrm{H}}}{\sqrt{L / C}}\left[\frac{1}{2} \frac{R}{\sqrt{L / C}} \cdot \frac{1}{\frac{\Delta L}{L}} \cos \omega_{1} t e^{-\alpha_{1} t}+\left(\sin \omega_{2} t-\frac{1}{2} \frac{R}{\sqrt{L / C}} \cdot \frac{1}{\frac{\Delta L}{L}} \cos \omega_{2} t\right) e^{-\alpha_{2} t}\right] \\
i_{1}(t)+i_{2}(t) \fallingdotseq \frac{E_{\mathrm{H}}}{\sqrt{L / C}}\left[\left(\sin \omega_{1} t+\frac{R}{\sqrt{L / C}} \cdot \frac{1}{\frac{\Delta L}{L}} \cos \omega_{1} t\right) e^{-\alpha_{1} t}+\left(\sin \omega_{2} t-\frac{R}{\sqrt{L / C}} \cdot \frac{1}{\frac{\Delta L}{L}} \cos \omega_{2} t\right) e^{-\alpha_{2} t}\right]
\end{array}\right\},
$$

where

$$
\begin{aligned}
& \alpha_{1} \fallingdotseq \frac{R}{L\left(1-\frac{\Delta L^{2}}{L^{2}}\right)}\left(1+\frac{\frac{\Delta L^{2}}{L^{2}}-\frac{1}{2} \frac{R^{2}}{L / C}}{\sqrt{\frac{\Delta L^{2}}{L^{2}}-\frac{R^{2}}{L / C}}}\right) \frac{\frac{\Delta L}{L} \gg \frac{R}{\sqrt{ } L / C}}{L} \rightarrow \frac{R}{L-\Delta L} \\
& \alpha_{2} \fallingdotseq \frac{R}{L\left(1-\frac{\Delta L^{2}}{L^{2}}\right)}\left(1-\frac{\frac{\Delta L^{2}}{L^{2}}-\frac{1}{2} \frac{R^{2}}{L / C}}{\sqrt{\frac{\Delta L^{2}}{L^{2}}-\frac{R^{2}}{L / C}}}\right) \longrightarrow \frac{R}{L+\Delta L} \\
& \omega_{1} \fallingdotseq \sqrt{\frac{1}{L C\left(1-\frac{\Delta L^{2}}{L^{2}}\right)}\left(1-\frac{1}{2} \frac{R^{2}}{L / C}+\sqrt{\left.\frac{\Delta L^{2}}{L^{2}}-\frac{R^{2}}{L / C}\right)-\alpha_{1}^{2}}\right.} \rightarrow \sqrt{\frac{1}{(L-\Delta L) C}} \\
& \omega_{2} \fallingdotseq \sqrt{\frac{1}{L C\left(1-\frac{\Delta L^{2}}{L^{2}}\right)}\left(1-\frac{1}{2} \frac{R^{2}}{L / C}-\sqrt{\left.\frac{\Delta L^{2}}{L^{2}}-\frac{R^{2}}{L / C}\right)-\alpha_{2}^{2}}\right.} \rightarrow \sqrt{\frac{1}{(L+\Delta L) C}}
\end{aligned} .
$$

When $\frac{\Delta L}{L}<\frac{R}{\sqrt{L / C}}$,

$$
\begin{aligned}
i_{1}(t) \fallingdotseq & \frac{1}{2} \frac{E_{\mathrm{H}}}{\sqrt{L / C}} \cdot \frac{1}{k}\left[\left((1+k) \sin \omega_{1}^{\prime} t-\frac{\frac{\Delta L}{R / \sqrt{L / C}}}{\left.\cos \omega_{1}^{\prime} t\right\} e^{-\alpha_{1}{ }^{\prime} t}}\right.\right. \\
& \left.-\left\{(1-k) \sin \omega_{2}^{\prime} t+\frac{\Delta L}{R / \sqrt{L / C}} \cos \omega_{2}^{\prime} t\right\} e^{-\alpha_{2}{ }^{\prime} t}\right]
\end{aligned}
$$


I. NAKAMURA: THE DECAY CHARACTERISTICS OF PIANO SOUND

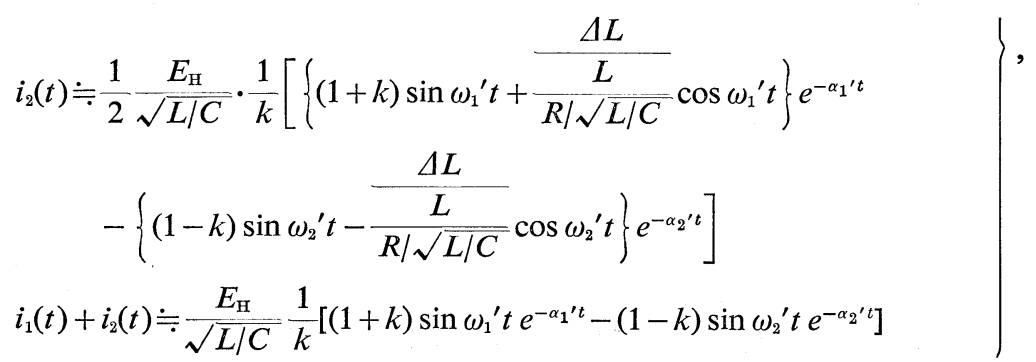

where

$$
\begin{aligned}
& \alpha_{1} \fallingdotseq \frac{R}{L\left(1-\frac{\Delta L^{2}}{L^{2}}\right)}\left(1+\frac{\sqrt{\frac{R^{2}}{L / C}-\frac{\Delta L^{2}}{L^{2}}}}{\frac{R}{\sqrt{L / C}}}\right) \frac{\frac{\Delta L}{L} \gg \frac{R}{\sqrt{L / C}}}{\sqrt{R^{2}}} \rightarrow \frac{2 R}{L} \\
& \alpha_{2}^{\prime} \fallingdotseq \frac{R}{L\left(1-\frac{\Delta L^{2}}{L^{2}}\right)}\left(1-\frac{\sqrt{\frac{R^{2}}{L / C}-\frac{\Delta L^{2}}{L^{2}}}}{\frac{R}{\sqrt{L / C}}}\right) \longrightarrow 0 \\
& \left.\omega_{1}^{\prime} \fallingdotseq \sqrt{\frac{1}{L C\left(1-\frac{\Delta L^{2}}{L^{2}}\right)}\left(1-\frac{1}{2} \frac{\Delta L^{2}}{L^{2}}+\frac{1}{2} \frac{\frac{R}{\sqrt{L / C}} \cdot \frac{\Delta L^{2}}{L^{2}}}{\sqrt{\frac{R^{2}}{L / C}-\frac{\Delta L^{2}}{L^{2}}}}\right)-\alpha_{1}^{\prime 2} \rightarrow \sqrt{\frac{1}{L C}-\left(\frac{2 R}{L}\right)^{2}}}\right\} .
\end{aligned}
$$

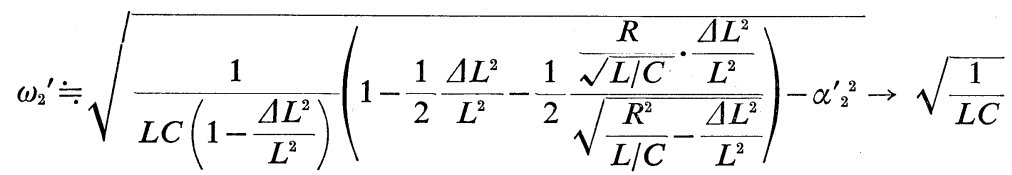

$$
\begin{aligned}
& k \fallingdotseq \sqrt{1-\frac{\Delta L^{2}}{L^{2}} \cdot \frac{L / C}{R^{2}}} \rightarrow 1 \\
& \text { Time(s) }
\end{aligned}
$$
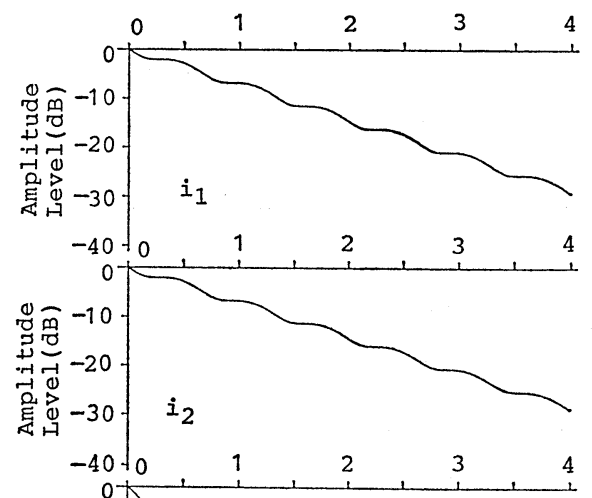

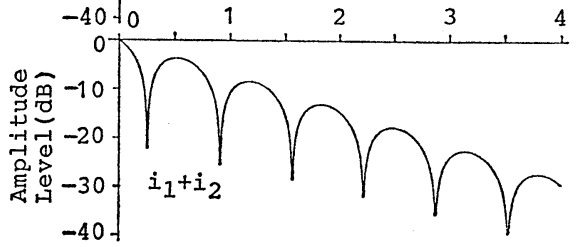

Fig. 4 Driving force when the two strings are loosely coupled.

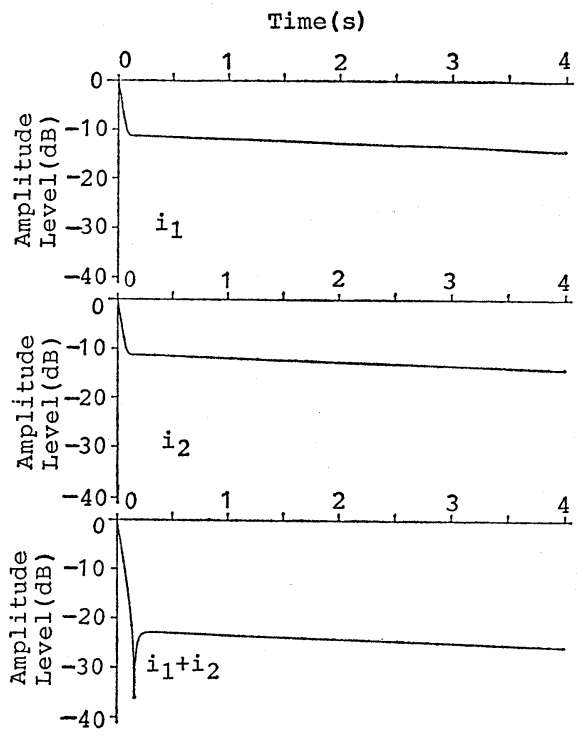

Fig. 5 Driving force when the two strings are closely coupled. 
For example, Fig. 4 shows the decay characteristics for $R / \sqrt{L / C}=2 \times 10^{-3}$ and $\Delta L / L=5.83 \times 10^{-3}$. Figure 5 shows the decay characteristics for $R / \sqrt{L / C}$ $=20 \times 10^{-3}$ and $\Delta L / L=2.90 \times 10^{-3}$. The curve $\left(i_{1}+i_{2}\right)$ in Fig. 4 shows a beat as the result of the difference between $\omega_{1}$ and $\omega_{2}$. The curve $\left(i_{1}+i_{2}\right)$ in Fig. 5 shows a local minimum value only once when the curve of $\alpha_{1}{ }^{\prime}$ is changed to the curve of $\alpha_{2}{ }^{\prime}$. Each partial shows various unique characteristics because of the variance in the impedance of the soundboard.
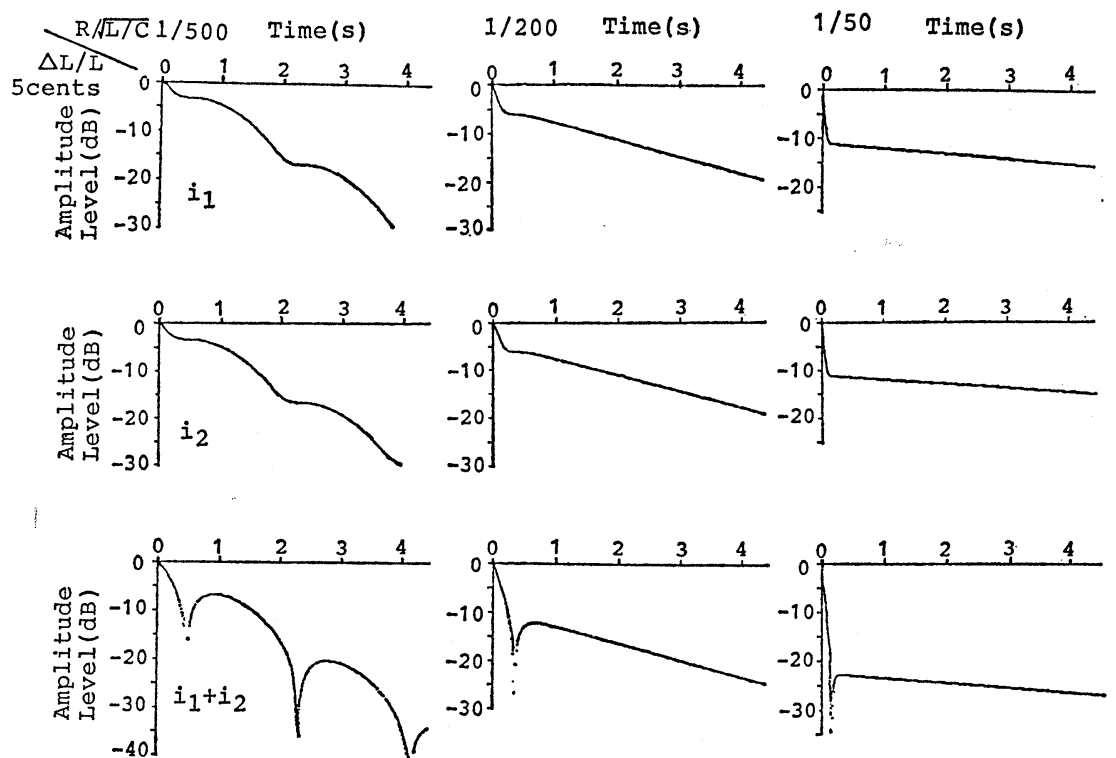

(a)

(b)

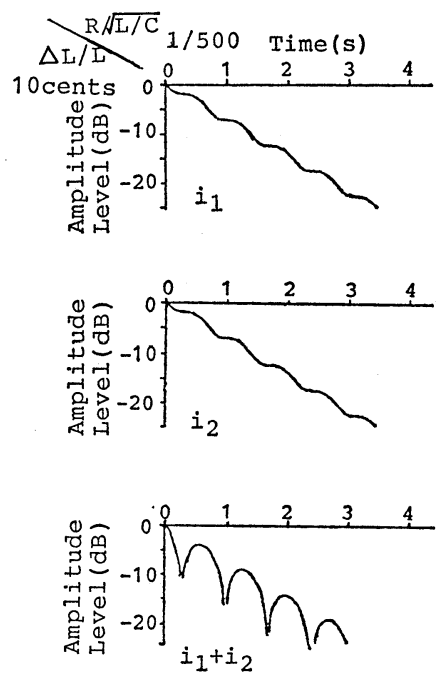

(a)

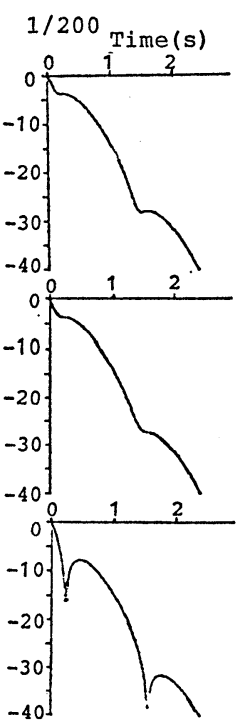

(b) (c)
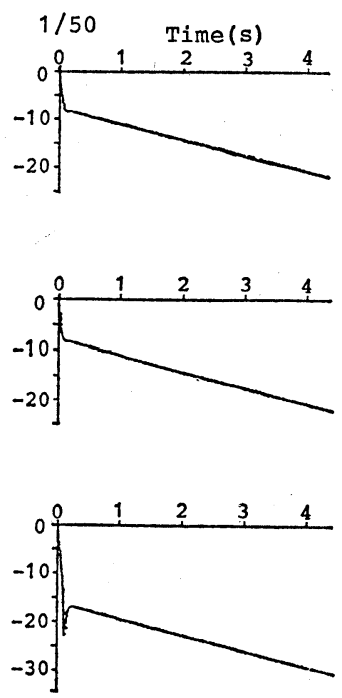

(c)

Fig. 6 Decay characteristics for various amounts of mistuning vs soundboard/string impedance ratios. 
The degree of mistuning is represented by $\Delta L / L$. The ratio of soundboard impedance to string impedance is expressed by $R / \sqrt{L / C}$. The degree of coupling between the two strings is dependent on the ratio of the above two constants. If the former is smaller than the latter, the two strings are strongly coupled to each other and a double decay characteristic results. If the relation between the two constants is reversed, the coupling is weak and a beat type decay characteristic results. The results show that the degree of tuning, and the string and the soundboard characteristics determine the decay characteristics.

\section{COMPUTER SIMULATION}

The following are the results of the computer simulation of the equivalent electrical circuit model. The initial condition is that when initial velocities are given, a constant voltage exists within the two condencers. The results of simulating various impedance ratios of the soundboard to the string, $R / \sqrt{L / C}$, when the degrees of mistuning are 5 cents and 10 cents, are shown in Fig. 6. These numerical values are not realistic, but they do not impose any problem for the theoretical model because the system is linear. The time variances of $i_{1}, i_{2}$ and $i_{1}+i_{2}$ in Fig. 6 show the decay characteristics of the envelope level of each amplitude. The theoretical values of $R / \sqrt{L / C}$ for Fig. 6 are $1 / 346$ when the level of mistuning is 5 cents, and $1 / 173$ in case of 10 cents. These figures clearly indicate that the transition from close coupling of two circuits to loose coupling appears at about $\Delta L / L=R / \sqrt{L / C}$, and that the loose coupling simply causes beats from the two vibrational systems, while the close coupling causes the separate prompt sound and after sound. These are changed by both the characteristic impedance ratio of the soundboard and the string, and the degree of mistuning.

Furthermore, the decay characteristics of $i_{1}, i_{2}$ and $i_{1}+i_{2}$ are shown in Fig. 7 for Fig. $6(\mathrm{~b})$ when the initial velocities with 1:0.8 ratio are given to the two strings. In this case, the amplitude of the string of the smaller velocity shows a slightly larger decay rate than the other one. However the degree of decay change is not as extreme as what Weinreich ${ }^{4}$ (1979) wrote. Figure 8 shows the wave forms of $i_{1}$ and $i_{2}$, in which the gradual shift from the in-phase to the anti-phase vibration is observed. The succeeding anti-phase state of the wave forms is shown in

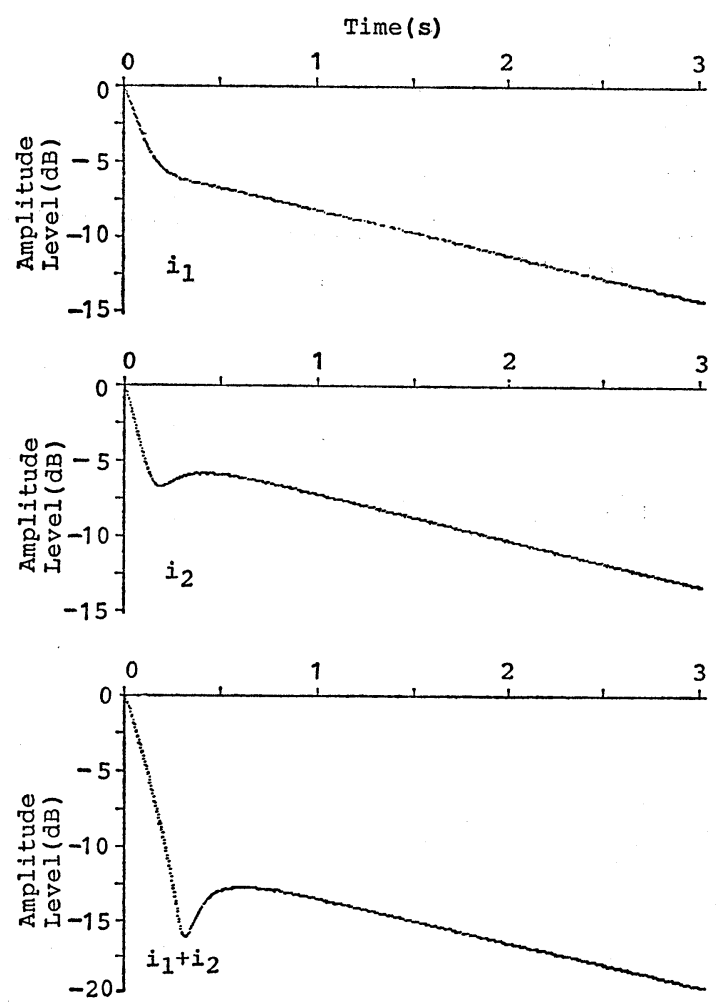

Fig. 7 Decay characteristics of $i_{1}, i_{2}$ and $i_{1}+i_{2}$ when the initial velocities with $1: 0.8$ ratio are given.

Fig. 9. The wave forms when they are stabilized are shown in Fig. 10. The wave forms remain slightly off as compared to the anti-phase state.

The following is a simple description of the three strings model. Figure 11 shows the decay characteristics when an additional string is inserted between the two strings with 10 cent mistuning as shown in Fig. $6(\mathrm{~b})^{\prime}$. It shows $i_{1}, i_{2}, i_{3}$ and $\left(i_{1}+i_{2}+i_{3}\right)$, when the strings are mistuned by 5 cents. Their prompt sounds and the after sounds are similar to that of Fig. 6(b) when the mistuning of two strings is by 5 cents, however, distinct beats appear during the aftersound. The results show that the outer two strings vibrate almost in-phase, while the middle string vibrates anti-phase. The driving force of the soundboard is almost in the same phase with the outer strings. These results agree with measured values and a simulation of a distributed-constant system. ${ }^{6)}$ 


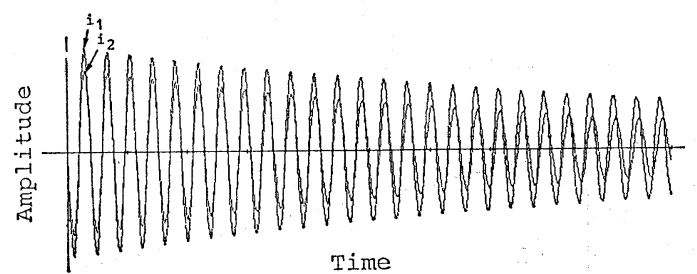

Fig. 8 Wave forms $i_{1}$ and $i_{2}$, in which the gradual shift from the in-phase vibration is observed.

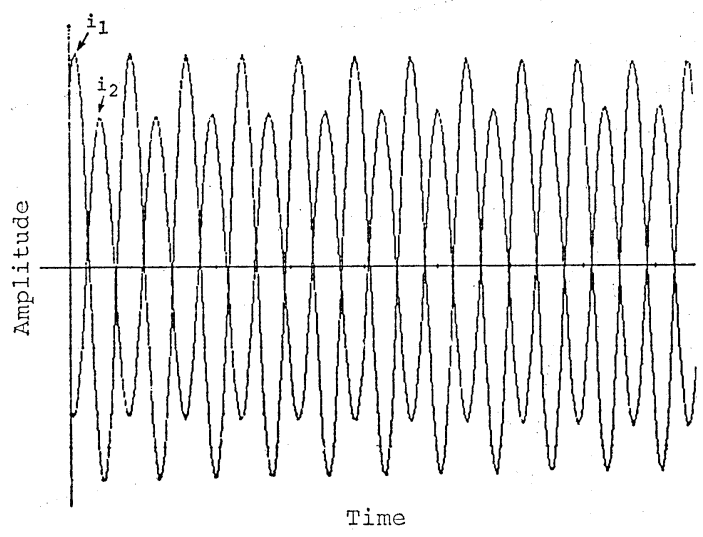

Fig. 9 Wave forms during the anti-phase period following the initial in-phase period.

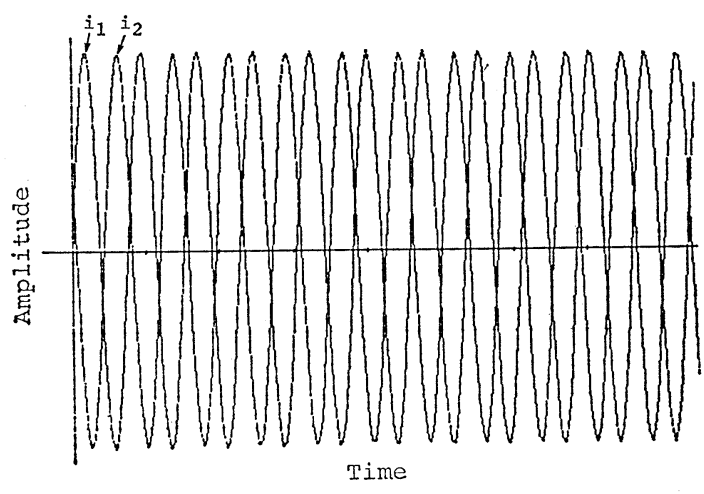

Fig. 10 Wave forms after stabilization.

\section{CONCLUSION}

The physical parameters which cause the prompt and after sounds of the piano were found to be the
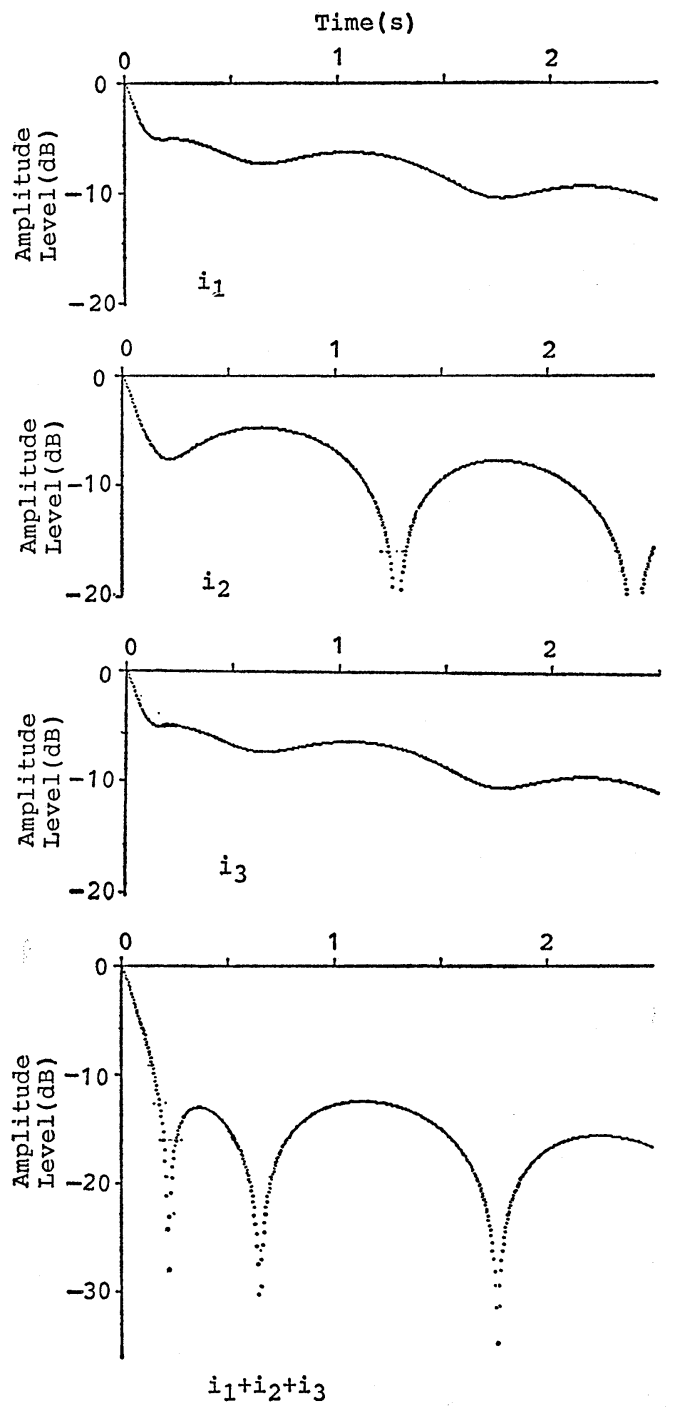

Fig. 11 Decay characteristics of $i_{1}, i_{2}, i_{3}$ and $\left(i_{1}+i_{2}+i_{3}\right)$ when the three strings are mistuned.

characteristic impedances of strings and the soundboard, and the degree of mistuning. Thus a fundamental understanding how the string and the soundboard, and the tuning work to control the piano sound has been achieved. The next step is to apply constants obtained by actual measurements to this theory in order to express the phenomenon in practical values. 


\section{NAKAMURA: THE DECAY CHARACTERISTICS OF PIANO SOUND}

\section{REFERENCES}

1) D. W. Martin, "Decay rates of piano tones," J. Acoust. Soc. Am. 19, 535-541 (1947).

2) R. E. Kirk, "Tuning preference for piano unison groups," J. Acoust. Soc. Am. 31, 1644-1648 (1959).

3) G. Weinreich, "Coupled piano strings," J. Acoust. Soc. Am. 62, 1474-1484 (1977).

4) G. Weinreich, "The coupled motions of piano strings," Sci. Am. 240, 94-102 (1979).

5) T. C. Hundley, H. Benioff, and D. W. Martin, "Factors contributing to the multiple rate of piano tone," J. Acoust. Soc. Am. 64, 1303-1309 (1978).

6) I. Nakamura, "Simulation of the sound production mechanism: Acoustical research on the piano (Part 2)," J. Acoust. Soc. Jpn. (J) 37, 65-75 (1981) (in Japanese).

7) K. Wogram, "Acoustical research on pianos, Part 1: Vibrational characteristics of the soundboard,"
Das Musikinstrument 24, 694-702, 776-782, 872880 (original German version in 1 bid 23, 380-404) (1981).

8) T. Yanagisawa, M. Morioka, K. Nakamura, and T. Miki, "Decay rate of partial vibration of piano string under the influence of soundboard," $\mathbf{J}$. Acoust. Soc. Jpn. (J) 40, $2-9$ (1984) (in Japanese).

9) I. Nakamura and S. Iwaoka, "Simulation of the vertical and horizontal vibrations on a piano string," Proc. Autumn Meet. Acoust. Soc. Jpn., 399-400 (1986) (in Japanese).

10) J. Meyer and A. Melka, "Messung und Darstellung des Ausklingverhaltens von Klavieren," Das Musikinstrument 32, 1049-1069 (1983).

11) I. Nakamura, S. Iwaoka, and T. Nakano, "Characteristics of piano tones in view of the spectrum study on the piano tone analysis, Part 1," Proc. Autumn Meet. Acoust. Soc. Jpn., 373-374 (1987) (in Japanese). 